\title{
Plaidoyer pour la bonne mise en œuvre des conventions TARMED
}

\author{
Andreas Haefelia, Roland Schwarz ${ }^{b}$ \\ a Dr, président de la commission paritaire de confiance Société des médecins d'Argovie - santésuisse \\ ${ }^{b}$ Dr, président de la commission paritaire cantonale des médecins Bâle-Campagne
}

\begin{abstract}
Possibilités et limites de la méthode statistique de screening dans le cadre du contrôle du caractère économique des prestations selon l'art. 56 al. 6 LAMal du point de vue d'un président de la commission paritaire de confiance cantonale (CPC).
\end{abstract}

\section{Contexte}

Bien que le corps médical ait toujours critiqué le caractère inadéquat des statistiques RSS et ANOVA de santésuisse employées jusqu'en 2016, le droit est toujours appliqué sur cette base - alors que l'erreur de prédiction atteint 30 à 50\% (Rapport final Polynomics SA, tableau 7 à la p. 38: www.fmh.ch/THÈMES/Tarifs ambulatoires/Groupes de travail/Groupe de travail EAE).

Suite à une décision du Parlement, l'art. 56 LAMal a été complété par un alinéa 6 qui est entré en vigueur le $1^{\text {er }}$ janvier 2013: «Les fournisseurs de prestations et les assureurs conviennent d'une méthode visant à contrôler le caractère économique des prestations.» Dans la convention du 23 août 2018 relative à la méthode statistique de screening dans le cadre du contrôle du caractère économique des prestations selon l'art. 56 al. 6 LAMal, les associations FMH, santésuisse et curafutura ont convenu d'une réglementation conventionnelle sous forme de onze articles concernant la méthode statistique de screening dans le cadre du contrôle du caractère économique des prestations (voir à ce sujet la convention: www.fmh. ch/THÈMES/Tarifs ambulatoires/Groupes de travail/ Groupe de travail EAE).

Cette convention a aussi son importance pour le travail des commissions paritaires de confiance cantonales (art. 17 de la convention-cadre TARMED LAMal), étant donné que celles-ci ont, entre autres, pour tâche la "vérification du bien-fondé de traitements médicaux par rapport aux critères d'efficacité, d'adéquation et d'économicité» selon l'annexe 6 à la convention-cadre TARMED LAMal.

En cas de litige entre fournisseurs de prestations et répondants des coûts dans le cadre de la vérification de la fourniture des prestations selon les critères d'effica- cité, d'adéquation et d'économicité d'après l'art. 32 LAMal, une procédure de conciliation se déroule, dans une première étape, dans le cadre de la commission paritaire de confiance cantonale et, dans un deuxième temps, devant le tribunal arbitral selon l'art. 89 LAMal. Par ailleurs, on notera qu'à l'art. 56 LAMal et dans la convention du 23 août 2018 relative à la méthode statistique de screening dans le cadre du contrôle du caractère économique des prestations selon l'art. 56 al. 6 LAMal, il est seulement question du "caractère économique des prestations». Les dispositions légales relatives à l'«efficacité» et à l'«adéquation» d'un traitement ne sont pas évoquées. Les critères de l'efficacité et de l'adéquation sont pourtant d'importance égale pour les commissions paritaires de confiance cantonales lors de l'appréciation de litiges.

Revenons à la convention du 23 août 2018 relative à la méthode statistique de screening dans le cadre du contrôle du caractère économique des prestations selon l'art. 56 al. 6 LAMal. Les deux premiers articles de la convention sont pertinents pour les commissions paritaires de confiance cantonales dans la mesure où meilleure cette méthode statistique de screening est, plus le nombre de médecins statistiquement hors normes sera petit. Ces médecins statistiquement hors normes seront cependant d'autant plus pertinents, avec moins de cas faux positifs ou faux négatifs, en ce qui concerne la fourniture de prestations supposée non économique. La méthode statistique de screening développée par Polynomics SA a été acceptée par santésuisse, curafutura et la FMH et est appliquée pour les données à compter de l'année 2017. L'article 3 de cette même convention prévoit que les données de la branche des assureurs-maladie (pool de données et pool tarifaire de SASIS SA) servent de données de base pour la méthode statistique de screening. 


\section{La difficulté de choisir le bon groupe de contrôle}

Le travail dans le cadre des procédures d'économicité devant le tribunal arbitral selon l'art. 89 LAMal montre cependant que les collectifs de comparaison, qui doivent être constitués dans ce cas, sont de qualité insuffisante et ne sont plus assez pertinents. L'expérience des dernières années dans le travail de la commission paritaire cantonale montre aussi que la base de données (p. ex. l'homogénéité des collectifs de comparaison) est de qualité insuffisante. Une amélioration de la qualité s'impose donc, car une base de données incomplète ne permet pas de réaliser des procédures d'économicité de bonne qualité.

Que s'est-il passé? Le pool de données de SASIS SA est alimenté par les données entrantes du registre des codes-créanciers. Jusqu'à présent, les numéros du registre des codes-créanciers étaient généralement attribués à des cabinets individuels ou des institutions regroupant des médecins de la même spécialité. Depuis un certain temps déjà, les numéros du registre des codes-créanciers sont attribués à des institutions dans lesquelles plusieurs médecins spécialistes de différentes disciplines facturent leurs prestations avec le même numéro du registre des codes-créanciers. Cette pratique ne permet pas d'atteindre, lors de la constitution de collectifs de comparaison sur la base du numéro du registre des codes-créancier, l'objectivité nécessaire par rapport à la discipline du médecin ou de l'institution sous le coup de la procédure. De plus, elle ne tient pas non plus compte de la sous-spécialisation croissante au sein d'un groupe de spécialistes, notamment en raison de la part élevée de médecins porteurs de deux titres de spécialistes. La comparaison statistique exigée ne fonctionne donc plus.

Cette erreur se répercute aussi directement dans le cadre de la nouvelle méthode statistique de screening, étant donné que la deuxième étape de l'analyse de régression tient compte des facteurs «emplacement du cabinet médical» et "groupe de spécialistes». Il faut donc s'attendre à une détérioration des résultats de la méthode statistique de screening.

Conclusion: la constitution adéquate du groupe de contrôle est essentielle pour l'application d'une méthode statistique. A l'heure actuelle, elle est entravée par les différentes structures organisationnelles des cabinets ainsi que par la fragmentation et la sous-spécialisation de la fourniture des prestations médicales. La constitution adéquate du groupe de contrôle doit pouvoir être vérifiée par les fournisseurs de prestations concernés, c'est-à-dire que ces derniers doivent pouvoir connaître au moins le nom des médecins figurant dans le collectif, mais aussi leur chiffre d'affaires (anonymisé) et leur éventail de prestations (pool tarifaire). Ici, il faut aussi tenir compte du fait que la nouvelle méthode statistique de screening ne couvre qu'une partie du contrôle du caractère économique des prestations selon l'art. 56 al. 6 LAMal. On relèvera en particulier que les indices de la méthode statistique de screening ne corrigent pas automatiquement et définitivement les particularités d'un cabinet. Seule une partie des particularités d'un cabinet est prise en compte par la nouvelle méthode statistique de screening, ce qui améliore certes la méthode statistique, mais ne remplace pas une appréciation individuelle en aval.

\section{Dans quels domaines la nouvelle méthode amène-t-elle des progrès et dans lesquels n'en amène-t-elle pas?}

La méthode a été testée avec un seuil de tolérance de $30 \%$ (indice 130) conformément à la jurisprudence actuelle. Lors de la discussion, les partenaires se sont mis d'accord pour conserver ce seuil. Une diminution p. ex. à 20\% (indice 120) ferait augmenter massivement le nombre de faux positifs compte tenu de la répartition statistique.

\section{Forces:}

- Morbidité via les groupes d'âge, $P C G^{1}$ et hospitalisation: les patients qui engendrent, en parallèle aux coûts de médicaments et d'hospitalisation trop élevés, des coûts plus élevés en cabinet sont identifiés. La liste des PCG utilisés doit être communiquée pour que le médecin puisse démontrer que sa patientèle diverge de la norme sur le plan clinique.

- Facteur d'incertitude: si certains patients au sein d'un cabinet engendrent des coûts élevés (généralement les $20 \%$ de patients les plus chers), cela provoque des fluctuations dans les coûts par groupe d'âge et est compensé par le facteur d'incertitude: le nouveau système est nettement moins sensible aux valeurs aberrantes, ce qui représente globalement la principale amélioration du système. La hauteur du facteur d'incertitude et le calcul utilisé pour le déterminer doivent être précisés.

\section{Faiblesses:}

- Les maladies (morbidité) qui engendrent des coûts de traitement élevés et qui ne sont pas identifiées par des coûts de médicaments et d'hospitalisation plus élevés provoquent des coûts élevés statistiquement non expliqués (p. ex.: patients souffrant de douleurs, soins palliatifs, patients souffrant de maladies psychiques). 
- Les prestations de nature différente ne sont pas détectées (p. ex. avec activité chirurgicale vs sans activité chirurgicale).

- L'activité dans deux disciplines n'est pas détectée (p. ex.: cardiologie + médecine de famille); cela pose notamment des problèmes lorsque la prestation supplémentaire doit être facturée avec les prestations de base.

- Collectif de patients particulier couches défavorisées, population issue de la migration, focalisation sur un groupe de maladies qui n'est pas représenté par les PCG (p. ex. VIH dans le groupe de spécialistes gynécologie et obstétrique).

\section{Conclusion au sujet de l'évaluation de la méthode:}

La nouvelle méthode statistique de screening représente une amélioration significative du système. Beaucoup de particularités des cabinets ne sont cependant pas prises en compte et doivent être examinées individuellement.

Comment peut-on remédier à ce problème? Les trois parties contractantes santésuisse, curafutura et la FMH ont probablement réfléchi plus loin, car elles n'auraient sinon pas prévu - à l'article 4 de la convention du 23 août 2018 relative à la méthode statistique de screening dans le cadre du contrôle du caractère économique des prestations selon l'art. 56 al. 6 LAMal - que «[...] les détails de la base de données, de la rectification des données/étapes de transformation et la spécification concrète de la méthode statistique mise en place [...] doivent être documentés et publiés conjointement [...]» C'est précisément sur ce point qu'il faut s'appuyer pour améliorer la méthode statistique de screening et entamer ce «travail de qualité». Cela aiderait toutes les parties impliquées dans le contrôle des prestations dans le cadre de la LAMal.

Enfin, la méthode statistique de screening identifie les médecins qui, en tenant compte des variables (âge, sexe, région, PCG, franchise et hospitalisation l'année précédente), présentent des coûts statistiquement hors normes. Cela ne prouve cependant pas que le médecin ne fournit et ne facture pas ses prestations conformément aux critères d'efficacité, d'adéquation et d'économicité, ou qu'il travaille de manière non économique.

\section{Avant le jugement vient l'enquête minutieuse}

L'appréciation de la question de savoir si un médecin fournit et facture des prestations de manière non économique doit, comme jusqu'ici, obligatoirement se faire, à la suite de la méthode statistique de screening, dans le cadre d'une appréciation individuelle du cas par santésuisse. On ne saurait donc admettre que santésuisse tente d'obtenir un remboursement de la part d'un médecin sur la seule base de ses valeurs d'indice plus élevées en argumentant que les valeurs d'indice de la nouvelle méthode statistique de screening corrigent automatiquement les particularités de son cabinet.

Etant donné qu'une méthode de screening se limite à des données simples et facilement accessibles, et qu'elle ne pourra donc jamais saisir toute la complexité de la problématique des prestations non économiques, elle ne constitue pas à elle seule un moyen de preuve approprié.

Comme la nouvelle méthode réduira fortement le nombre de médecins statistiquement hors normes, on pourra et devra consacrer davantage de temps à l'analyse approfondie et à l'appréciation individuelle. La Suisse ferait bien de s'adapter aux normes en vigueur dans les pays voisins et de TOUJOURS demander une évaluation individuelle en cas de procédure judiciaire.

La poursuite du développement du contrôle du caractère économique des prestations devrait donc impliquer, d'une part, le perfectionnement de la méthode discutée dans cet article et, d'autre part, la définition partenariale de la méthode d'évaluation individuelle. 